\title{
QUANTUM ITÔ B*-ALGEBRAS, THEIR CLASSIFICATION AND DECOMPOSITION
}

\author{
V. P. BELAVKIN \\ Mathematics Department, University of Nottingham \\ Nottingham, NGr 2RD, UK \\ E-mail: vpb@maths.nott.ac.uk
}

\begin{abstract}
A simple axiomatic characterization of the general (infinite dimensional, noncommutative) Itô algebra is given and a pseudo-Euclidean fundamental representation for such algebra is described. The notion of Itô $\mathrm{B}^{*}$-algebra, generalizing the $\mathrm{C}^{*}$-algebra, is defined to include the Banach infinite dimensional Itô algebras of quantum Brownian and quantum Lévy motion, and the $\mathrm{B}^{*}$-algebras of vacuum and thermal quantum noise are characterized. It is proved that every Itô algebra is canonically decomposed into the orthogonal sum of quantum Brownian (Wiener) algebra and quantum Lévy (Poisson) algebra. In particular, every quantum thermal noise is the orthogonal sum of a quantum Wiener noise and a quantum Poisson noise as it is stated by the Lévy-Khinchin Theorem in the classical case.
\end{abstract}

1. Introduction: Itô $\star$-algebras. The classical stochastic calculus developed by Itô, and its quantum stochastic analog, given by Hudson and Parthasarathy (HP) in [1], can be unified in the $\star$-algebraic approach to the operator integration in Fock space [2], in which the classical and quantum calculi become represented as two extreme commutative and completely noncommutative cases of a generalized Itô calculus.

On the axiomatic level the generalized Itô algebra was defined in [3] by a family of stochastic differentials $\mathrm{d} \Lambda(t, a), a \in \mathfrak{a}$ with respect to $t \in \mathbb{R}_{+}$, generating an associative, but in general noncommutative $\star$-algebra: $\mathrm{d} \Lambda(a)^{\dagger}=\mathrm{d} \Lambda\left(a^{\star}\right)$,

$$
\mathrm{d} \Lambda(a)^{\dagger} \mathrm{d} \Lambda(a)=\mathrm{d} \Lambda\left(a^{\star} a\right), \quad \sum \lambda_{i} \mathrm{~d} \Lambda\left(a_{i}\right)=\mathrm{d} \Lambda\left(\sum \lambda_{i} a_{i}\right)
$$

with given mean values $\langle\mathrm{d} \Lambda(t, a)\rangle=l(a) \mathrm{d} t$, absolutely continuous with respect to $\mathrm{d} t=$ $\mathrm{d} \Lambda(t, \theta)$. Here the indexing $\star$-semigroup $\mathfrak{a}$ is extended to a complex, in general infinite dimensional linear space, parametrizing the Itô algebra, with an involution $a \mapsto a^{\star} \in \mathfrak{a}$, $a^{\star \star}=a$ and death $\theta=\theta^{\star} \in \mathfrak{a}$, a self-adjoint annihilator $\mathfrak{a} \theta=0$, corresponding to

This work is supported in part by the State Committee for Scientific Research, under project 2P03P 12911.

The paper is in final form and no version of it will be published elsewhere. 
$\mathrm{d} \Lambda(t, \mathfrak{a}) \mathrm{d} t=0$, and $l: \mathfrak{a} \rightarrow \mathbb{C}$ is a positive $l\left(a^{\star} a\right) \geq 0$ linear $*$-functional, $l\left(a^{\star}\right)=l(a)^{*}$, normalized as $l(\theta)=1$ due to the determinism $\langle\mathrm{d} t\rangle=\mathrm{d} t$.

The functional $l$ defines the fundamental representation $\boldsymbol{i}: a \mapsto \boldsymbol{a}=\left(a_{\nu}^{\mu}\right)_{\nu=+, \bullet}^{\mu=-\mathfrak{\bullet}}$ of $\mathfrak{a}$ in terms of the quadruples

$$
a_{\bullet}^{\bullet}=i(a), \quad a_{+}^{\bullet}=k(a), \quad a_{\bullet}^{-}=k^{*}(a), \quad a_{+}^{-}=l(a),
$$

where $i(a)=i\left(a^{\star}\right)^{\dagger}$ is the GNS representation $k(a b)=i(a) k(b)$ of $\mathfrak{a}$ in the Hilbert space $\mathcal{H} \ni k(b), b \in \mathfrak{a}$ of the Kolmogorov decomposition $l\left(a^{\star} b\right)=k(a)^{\dagger} k(b)$, and $k^{*}(a)=k\left(a^{\star}\right)^{\dagger}$. In these tensor notations $a_{\nu}^{\mu}=i_{\nu}^{\mu}(a), \mu=-, \bullet, \nu=+, \bullet$ the composition properties $i(a) i(b)=i(a b), k^{*}(a) i(b)=k^{*}(a b)$ together with the already mentioned $i(a) k(b)=k(a b)$, $k^{*}(a) k(b)=l(a b)$, can be written in the form of the multiplicativity $\mathrm{i}(a b)=\boldsymbol{a} \bullet \boldsymbol{b}$ with respect to the convolution

$$
\boldsymbol{a} \bullet \boldsymbol{b}=\left(a_{\bullet}^{\mu} b_{\nu}^{\bullet}\right)_{\nu=+, \bullet}^{\mu=-\bullet}
$$

of the components $a_{\nu}$ and $b^{\mu}$ over the common index values $\mu=\bullet=\nu$ only. One can also use the convention $a_{-}^{\mu}=0=a_{\nu}^{+}$of the tensor notations (2) to extend the quadruples $\boldsymbol{a}=\boldsymbol{i}(a)$ to the matrices $\mathbf{a}=\left[a_{\nu}^{\mu}\right]_{\nu=-, \bullet,+}^{\mu=-,+}$, in which (3) is simply given as $\mathbf{i}(a b)=\mathbf{a b}$ in terms of the usual product of the matrices $\mathbf{a}=\mathbf{i}(a)$ and $\mathbf{b}=\mathbf{i}(b)$. But the involution $a \mapsto a^{\star}$, which is represented in terms of the quadruples as the Hermitian conjugation $\boldsymbol{i}\left(a^{\star}\right)=\left(a_{-\mu}^{-\nu \dagger}\right)_{\nu=-, \bullet}^{\mu=-\bullet}$, where $-(-)=+,-\bullet=\bullet,-(+)=-$, is given by the adjoint matrix $\mathbf{g a}^{\dagger} \mathbf{g}=\mathbf{i}\left(a^{\star}\right)$ w.r.t. the pseudo-Hilbert (complex Minkowski) metrics $\mathbf{g}=\left[\delta_{-\nu}^{\mu}\right]_{\nu=-, \bullet,+}^{\mu=-,+}$ as it was noted in $[2,3]$.

It was proved in $[3,4]$ that any (classical or quantum) stochastic noise described by a process $t \in \mathbb{R}_{+} \mapsto \Lambda(t, a), a \in \mathfrak{a}$ with independent increments $\mathrm{d} \Lambda(t, a)=\Lambda(t+\mathrm{d} t, a)-$ $\Lambda(t, a)$, forming an Itô $*$-algebra, can be represented in the (symmetric) Fock space $\mathfrak{F}$ over the space of $\mathcal{H}$-valued square-integrable functions on $\mathbb{R}_{+}$as $\Lambda(t, a)=a_{\nu}^{\mu} \Lambda_{\mu}^{\nu}(t)$. Here

$$
a_{\nu}^{\mu} \Lambda_{\mu}^{\nu}(t)=a_{\bullet}^{\bullet} \Lambda_{\bullet}^{\bullet}(t)+a_{+}^{\bullet} \Lambda_{\bullet}^{+}(t)+a_{\bullet}^{-} \Lambda_{-}^{\bullet}(t)+a_{+}^{-} \Lambda_{-}^{+}(t)
$$

is the canonical decomposition of $\Lambda$ into the exchange $\Lambda_{\bullet}^{\bullet}$, creation $\Lambda_{\bullet}^{+}$, annihilation $\Lambda_{-}^{\bullet}$ and preservation (time) $\Lambda_{-}^{+}=t$ I operator-valued processes of the HP quantum stochastic calculus, having the mean values $\left\langle\Lambda_{\mu}^{\nu}(t)\right\rangle=t \delta_{+}^{\nu} \delta_{\mu}^{-}$with respect to the vacuum state in $\mathfrak{F}$. Thus the parametrizing algebra $\mathfrak{a}$ of the processes with independent increments can be always represented by a $\star$-subalgebra $\{\boldsymbol{i}(a) \mid a \in \mathfrak{a}\}$ of the non-unital algebra $\mathfrak{b}(\mathcal{H})$ of all quadruples $\boldsymbol{a}=\left(a_{\nu}^{\mu}\right)_{\nu=+, \bullet}^{\mu=-\bullet}$ with the product (3) and the unique death, given by the quadruple $\theta=\left(\delta_{-}^{\mu} \delta_{\nu}^{+}\right)_{\nu=+, \bullet}^{\mu=-} \bullet$ having the only nonzero element $\theta_{+}^{-}=1$. Here $a_{\nu}^{\mu}: \mathcal{H}_{\nu} \rightarrow \mathcal{H}_{\mu}$ are the linear operators on $\mathcal{H}_{\bullet}=\mathcal{H}, \mathcal{H}_{+}=\mathbb{C}=\mathcal{H}_{-}$, having the adjoints $a_{\nu}^{\mu \dagger}: \mathcal{H}_{\mu} \rightarrow \mathcal{H}_{\nu}$, which define the natural involution $\boldsymbol{a} \mapsto \boldsymbol{a}^{\star}$ on $\mathfrak{b}(\mathcal{H})$, given by $a_{-\nu}^{\star \mu}=a_{-\mu}^{\nu \dagger}$.

The simplest, one-dimensional Itô algebra generated by the nonstochastic differential of the deterministic process $\Lambda(t, \theta)=t$ I corresponds to the Newton differential calculus $(\mathrm{d} t)^{2}=0$. It is described by the smallest Itô algebra $\mathfrak{a}=\mathbb{C} \theta$ with $l(a)=\alpha \in \mathbb{C}$ for $a=\alpha \theta$ and the nilpotent multiplication $\alpha^{\star} \alpha=0$ with $\alpha^{\star}=\bar{\alpha}$ representing the classical non-stochastic calculus in $\mathcal{H}=\{0\}$.

The classical stochastic calculus $\mathrm{d} w_{i} \mathrm{~d} w_{k}=\delta_{k}^{i} \mathrm{~d} t$ for the Itô differentials $\mathrm{d} w_{i}, i=$ $1, \ldots, d$ of the standard Wiener processes $w_{i}(t)$ generates the $d+1$ dimensional Itô algebra as the second order nilpotent algebra $\mathfrak{a}$ of pairs $a=(\alpha, \eta), \eta \in \mathbb{C}^{d}$ with $\theta=(1,0)$ and 
$a^{\star} a=\left(\|\eta\|^{2}, 0\right)$ for $a^{\star}=\left(\bar{\alpha}, \eta^{\star}\right)$, where $\eta^{\star}=\left(\bar{\eta}^{i}\right)$ is the complex conjugation of $\eta=\left(\eta^{i}\right)$ and $\|\eta\|^{2}=\sum_{i=1}^{d}\left|\eta^{i}\right|^{2}$. The GNS representation associated with $l(a)=\alpha$ is given by $a_{+}^{-}=\alpha, a_{i}^{-}=\eta^{i}=a_{+}^{i}, a_{\bullet}:=0$ in $\mathcal{H}=\mathbb{C}^{d}$, and the operator representation of $\mathfrak{a}$ in Fock space is defined by the forward differentials of $\Lambda(t, a)=\alpha t \mathrm{I}+\sum_{i=1}^{d} \eta^{i} \mathrm{~W}_{i}(t)$, where $\mathrm{W}_{i}(t)=\Lambda_{-}^{i}(t)+\Lambda_{i}^{+}(t)$ are the operator representations of the processes $w_{i}(t)$ with respect to the vacuum state in $\mathfrak{F}$.

The unital $\star$-algebra of complex vectors $\zeta=\left(\zeta^{i}\right)$ with component-vise multiplication $\zeta^{\star} \zeta=\left(\left|\zeta^{i}\right|^{2}\right)=|\zeta|^{2}$ can be embedded into the $d+1$-dimensional Itô algebra $\mathfrak{a}$ of the pairs $a=(\alpha, \zeta), \zeta \in \mathbb{C}^{d}$ with $\theta=(1,0)$ and $a^{\star} a=\left(\|\zeta\|^{2},|\zeta|^{2}\right)$ by $\alpha=\sum_{i=1}^{d} \zeta^{i}$. The GNS representation of $\mathfrak{a}$, associated with $l(a)=\alpha$, is given by $a_{+}^{-}=\alpha, a_{i}^{-}=\zeta^{i}=a_{+}^{i}$, $a_{k}^{i}=\delta_{k}^{i} \zeta^{i}$, and the Fock space representation is defined by $\Lambda(t, a)=\alpha t \mathrm{I}+\sum_{i=1}^{d} \zeta^{i} \mathrm{M}_{i}(t)$, where $\mathrm{M}_{i}(t)=\Lambda_{i}^{i}(t)+\mathrm{W}_{i}(t)$ are the operator representations in $\mathfrak{F}$ of the standard Poisson processes $n_{i}(t)=m_{i}(t)+t$, compensated by their vacuum mean values $\left\langle n_{i}(t)\right\rangle=t$, with $\mathrm{d} n_{i} \mathrm{~d} n_{k}=\delta_{k}^{i} \mathrm{~d} n_{k}$.

Note that any two-dimensional Itô $\star$-algebra $\mathfrak{a}$ is commutative as $\theta a=0=a \theta$ for any other element $a \neq \theta$ of a basis $\{a, \theta\}$ in $\mathfrak{a}$. Moreover, each such algebra is either of the Wiener or of the Poisson type as it is either second order nilpotent, or contains a unital one-dimensional subalgebra. Thus our results on the classification of Itô $\star$-algebras will be nontrivial only in the higher dimensions of $\mathfrak{a}$. The well known Levy-Khinchin classification of the classical noise can be reformulated in purely algebraic terms as the decomposability of any commutative Itô algebra into the Newton, Wiener (Brownian) and Poisson (Lévy) orthogonal components. In the general case we shall show that every Itô $\star$-algebra is also decomposable into the Newton, a quantum Brownian, and a quantum Lévy orthogonal components.

Let us first consider the following class of strictly non-commutative vacuum Itô algebras which have no classical analogs, and then a more ordinary class of thermal Itô algebras as they were defined in $[3,4]$ by a generalization of classical Wiener and Poisson noise algebras.

2. Itô $\mathbf{B}^{*}$-algebra of vacuum noise. Let $\mathfrak{h}$ be a Hilbert space of ket-vectors $x$ with scalar product $\langle x \mid x\rangle$ and $\mathcal{A} \subseteq \mathcal{B}(\mathfrak{h})$ be a $\mathrm{C}^{*}$-algebra, represented on $\mathfrak{h}$ by the operators $\mathcal{A} \ni A: x \mapsto A x$ with $\left\langle A^{\dagger} x \mid x\right\rangle=\langle x \mid A x\rangle$. We denote by $\mathfrak{h}^{*}$ the dual Hilbert space of bra-vectors $y=z^{*}, z \in \mathfrak{h}$ with the scalar product $\left\langle y \mid x^{*}\right\rangle=y x=\left\langle y^{*} \mid x\right\rangle$ given by inverting anti-linear isomorphism $\mathfrak{h}^{*} \ni y \mapsto y^{*} \in \mathfrak{h}$, and the dual representation of $\mathcal{A}$ as the right representation $A^{\prime}: y \mapsto y A, y \in \mathfrak{h}^{*}$, given by $(y A) x=y(A x)$ such that $\left\langle y A^{\dagger} \mid y\right\rangle=\langle y \mid y A\rangle$ on $\mathfrak{h}^{*}$. Then the direct sum $\mathcal{K}=\mathfrak{h} \oplus \mathfrak{h}^{*}$ becomes a two-sided $\mathcal{A}$-module

$$
A \xi=A x, \quad \xi A=y A, \quad \forall \xi=x \oplus y,
$$

with the flip-involution $(x \oplus y)^{\star}=y^{*} \oplus x^{*}$ and two scalar products

$$
\left\langle x \oplus y^{\prime} \mid x^{\prime} \oplus y\right\rangle_{+}=\left\langle x \mid x^{\prime}\right\rangle, \quad\left\langle x \oplus y^{\prime} \mid x^{\prime} \oplus y\right\rangle^{-}=\left\langle y^{\prime} \mid y\right\rangle .
$$

The space $\mathfrak{a}=\mathbb{C} \oplus \mathcal{K} \oplus \mathcal{A}$ of triples $a=(\alpha, \xi, A)$ becomes an Itô $\star$-algebra with respect to the non-commutative product

$$
a^{\star} a=\left(\langle\xi \mid \xi\rangle_{+}, \xi^{\star} A+A^{\dagger} \xi, A^{\dagger} A\right), \quad a a^{\star}=\left(\langle\xi \mid \xi\rangle^{-}, A \xi^{\star}+\xi A^{\dagger}, A A^{\dagger}\right),
$$


where $(\alpha, \xi, A)^{\star}=\left(\bar{\alpha}, \xi^{\star}, A^{\dagger}\right)$, with death $\theta=(1,0,0)$ and $l(\alpha, \xi, A)=\alpha$. Obviously $a^{\star} a \neq a a^{\star}$ if $\|\xi\|_{+}=\|x\| \neq\|y\|=\|\xi\|^{-}$even if the operator algebra $\mathcal{A}$ is commutative, $A^{\dagger} A=A A^{\dagger}$. It is separated by four semi-norms

$$
\|a\|=\|A\|,\|a\|_{+}=\|x\|,\|a\|^{-}=\|y\|,\|a\|_{+}^{-}=|\alpha|,
$$

and is jointly complete as $a=(\alpha, x \oplus y, A) \in \mathfrak{a}$ have independent components from the Banach spaces $\mathbb{C}, \mathfrak{h}, \mathfrak{h}^{*}$ and $\mathcal{A}$.

We shall call such Banach Itô algebra the vacuum algebra as $l\left(a^{\star} a\right)=0$ for any $a \in \mathfrak{a}$ with $\xi \in \mathfrak{h}^{*}$ (the Hudson-Parthasarathy algebra $\mathfrak{a}=\mathfrak{b}(\mathfrak{h})$ if $\mathcal{A}=\mathcal{B}(\mathfrak{h})$ ). Every closed Itô subalgebra $\mathfrak{a} \subseteq \mathfrak{b}(\mathcal{H})$ of the HP algebra $\mathfrak{b}(\mathcal{H})$ equipped with four norms (8) on a Hilbert space $\mathcal{H}$ is called the operator Itô $\mathrm{B}^{*}$-algebra.

If the algebra $\mathcal{A}$ is completely degenerated on $\mathfrak{h}, \mathcal{A}=\{0\}$, the Itô algebra $\mathfrak{a}$ is nilpotent of second order, and contains only the two-dimensional subalgebras of Wiener type $\mathfrak{b}=$ $\mathbb{C} \oplus \mathbb{C} \oplus\{0\}$ generated by an $a=(\alpha, x \oplus y, 0)$ with $\|x\|=\|y\|$. Every closed Itô subalgebra $\mathfrak{b} \subseteq \mathfrak{a}$ of the HP B*-algebra $\mathfrak{a}=\mathfrak{b}(\mathcal{H})$ is called the B*-Itô algebra of a vacuum Brownian motion if it is defined by a $\star$-invariant direct sum $\mathcal{G}=\mathcal{G}_{+} \oplus \mathcal{G}^{-} \subseteq \mathcal{K}$ given by a Hilbert subspace $\mathcal{G}_{+} \subseteq \mathcal{H}, \mathcal{G}^{-}=\mathcal{G}_{+}^{*}$ and $\mathcal{A}=\{0\}$.

In the case $I \in \mathcal{A}$ the algebra $\mathcal{A}$ is not degenerated and contains also the vacuum Poisson subalgebra $\mathbb{C} \oplus\{0\} \oplus \mathbb{C} I$ of the total quantum number on $\mathfrak{h}$, and other Poisson two-dimensional subalgebras, generated by $a=(\alpha, x \oplus y, I)$ with $y=e^{i \phi} x^{*}$. We shall call a closed Itô subalgebra $\mathfrak{c} \subseteq \mathfrak{a}$ of the HP B*-algebra $\mathfrak{a}=\mathfrak{b}(\mathcal{H})$ the B*-algebra of a vacuum Lévy motion if it is given by a direct sum $\mathcal{E}=\mathcal{E}_{+} \oplus \mathcal{E}^{-} \subseteq \mathcal{K}$ with $\mathcal{E}^{-}=\mathcal{E}_{+}^{*}$ and a $*$-subalgebra $\mathcal{A} \subseteq \mathcal{B}(\mathcal{H})$ nondegenerated on the subspace $\mathcal{E}_{+} \subseteq \mathcal{H}$.

We shall see that the general vacuum $B^{*}$-algebra $\mathfrak{a}$, which is characterized by the condition $\mathfrak{n}_{+}^{\perp}=\mathfrak{n}^{-}$, where

$$
\mathfrak{n}^{-}=\left\{b \in \mathfrak{a}:\|b\|^{-}=0\right\}, \quad \mathfrak{n}_{+}=\left\{c \in \mathfrak{a}:\|c\|_{+}=0\right\},
$$

and $\mathfrak{n}_{+}^{\perp}$ is the right orthogonal complement to $\mathfrak{n}_{+}$, can be represented as an operator vacuum Itô $\mathrm{B}^{*}$-algebra.

THEOREM 1. Every vacuum $B^{*}$-algebra can be decomposed into an orthogonal sum $\mathfrak{a}=\mathfrak{b}+\mathfrak{c}, \mathfrak{b} c=\{0\}$ of the Brownian vacuum $B^{*}$-algebra $\mathfrak{b}$ and the Lévy vacuum $B^{*}$-algebra c.

Proof. This decomposition is uniquely defined for all $a=(\alpha, \xi, A)$ by $a=\alpha \theta+b+c$, with $b=(0, \eta, 0), c=(0, \zeta, A), \eta=P x \oplus y P \in \mathcal{G}, \zeta=\xi-\eta \in \mathcal{E}$, where $P=P^{\dagger}$ is the maximal projector in $\mathfrak{h}$, for which $\mathcal{A} P=\{0\}, \mathcal{G}_{+}=P \mathfrak{h}$, and $\mathcal{E}_{+}=\mathcal{G}_{+}^{\perp}$.

3. Itô $\mathbf{B}^{*}$-algebra of thermal noise. Let $\mathcal{D}$ be a left Tomita $\star$-algebra [5] with respect to a Hilbert norm $\|\xi\|_{+}=0 \Rightarrow \xi=0$, and thus a right pre-Hilbert $\star$-algebra with respect to $\|\xi\|^{-}=\left\|\xi^{\star}\right\|_{+}$. This means that $\mathcal{D}$ is a complex pre-Hilbert space with continuous left (right) multiplications $C: \zeta \mapsto \xi \zeta\left(C^{\prime}: \eta \mapsto \eta \xi\right)$ w.r.t. $\|\cdot\|_{+}$(w.r.t. $\left.\|\cdot\|^{-}\right)$ of the elements $\zeta, \eta \in \mathcal{D}$ respectively, defined by an associative product in $\mathcal{D}$, and the involution $\mathcal{D} \ni \xi \mapsto \xi^{\star} \in \mathcal{D}$ such that

$$
\left\langle\eta \zeta^{\star} \mid \xi\right\rangle^{-}=\langle\eta \mid \xi \zeta\rangle^{-}, \quad\left\langle\eta^{\star} \zeta \mid \xi\right\rangle_{+}=\langle\zeta \mid \eta \xi\rangle_{+} \quad \forall \xi, \zeta, \eta \in \mathcal{D}
$$




$$
\left\langle\eta \mid \xi^{\star}\right\rangle^{-}=\left\langle\xi \mid \eta^{\sharp}\right\rangle^{-}, \quad\left\langle\zeta \mid \xi^{\star}\right\rangle_{+}=\left\langle\xi \mid \zeta^{b}\right\rangle_{+} \quad \forall \eta \in \mathcal{D}^{-}, \zeta \in \mathcal{D}_{+} .
$$

Here $\left\langle\eta \mid \zeta^{\star}\right\rangle^{-}=\left\langle\eta^{\star} \mid \zeta\right\rangle_{+}$is the right scalar product, $\mathcal{D}_{+}=\mathcal{D}_{+}^{b}$ is a dense domain for the left adjoint involution $\zeta \mapsto \zeta^{b}, \zeta^{b b}=\zeta$, and $\mathcal{D}^{-}=\mathcal{D}_{+}^{\star}$ is the invariant domain for the right adjoint involution $\eta \mapsto \eta^{\sharp},\left(\eta^{\sharp} \eta\right)^{\sharp}=\eta^{\sharp} \eta$ such that $\zeta^{b \star}=\zeta^{\star \natural}, \eta^{\sharp \star}=\eta^{\star b}$.

Since the adjoint operators $C^{\dagger} \zeta=\xi^{\star} \zeta, \eta C^{\dagger}=\eta \xi^{\star}$ are also given by the multiplications, they are bounded:

$$
\|\xi\|=\sup \left\{\|\xi \zeta\|_{+}:\|\zeta\|_{+} \leq 1\right\}=\sup \left\{\|\eta \xi\|^{-}:\|\eta\|^{-} \leq 1\right\}<\infty .
$$

Note that we do not require the sub-space $\mathcal{D D} \subseteq \mathcal{D}$ of all products $\eta \zeta, \eta, \zeta \in \mathcal{D}$ to be dense in $\mathcal{D}$ w.r.t. any of two Hilbert norms on $\mathcal{D}$, but it is always dense w.r.t. the operator semi-norm (12) on $\mathcal{D}$. Hence the operator $\dagger$-algebra $\mathcal{C}=\{C: \mathcal{D} \ni \zeta \mapsto \xi \zeta \mid \xi \in \mathcal{D}\}$ w.r.t. the left scalar product, which is also represented on the $\mathcal{D} \ni \eta$ equipped with $\langle\cdot \mid \cdot\rangle^{-}$by the right multiplications $\eta C=\eta \xi, \xi \in \mathcal{D}$, can be degenerated on $\mathcal{D}$.

Thus the direct sum $\mathfrak{a}=\mathbb{C} \oplus \mathcal{D}$ of pairs $a=(\alpha, \xi)$ becomes an Itô $\star$-algebra with the product

$$
a^{\star} a=\left(\langle\xi \mid \xi\rangle_{+}, \xi^{\star} \xi\right), \quad a a^{\star}=\left(\langle\xi \mid \xi\rangle^{-}, \xi \xi^{\star}\right),
$$

where $(\alpha, \xi)^{\star}=\left(\bar{\alpha}, \xi^{\star}\right)$, with death $\theta=(1,0)$ and $l(\alpha, \xi)=\alpha$. Obviously $a^{\star} a \neq a a^{\star}$ if the involution $a \mapsto a^{\star}$ is not isometric w.r.t. any of two Hilbert norms even if the algebra $\mathcal{D}$ is commutative. It is a Banach algebra if its normed $\star$-algebra $\mathcal{D}$ is complete jointly w.r.t. three norms, and is separated by the semi-norms

$$
\|a\|=\|\xi\|,\|a\|^{-}=\|\xi\|^{-},\|a\|_{+}=\|\xi\|_{+},\|a\|_{+}^{-}=|\zeta| .
$$

We shall call such complete Itô algebra the thermal $\mathrm{B}^{*}$-algebra as $l\left(a^{\star} a\right)=\|\xi\|_{+}^{2} \neq 0$ for any $a \in \mathfrak{a}$ with $\xi \neq 0$. If $\zeta \eta=0$ for any $\zeta, \eta \in \mathcal{D}$, it is the Itô $\mathrm{B}^{*}$-algebra of thermal Brownian motion. A thermal $\mathrm{B}^{*}$-subalgebra $\mathfrak{b} \subseteq \mathfrak{a}$ with such trivial product is given by any involutive pre-Hilbert $\star$-invariant two-normed subspace $\mathcal{G} \subseteq \mathcal{D}$ which is closed w.r.t. the Hilbert sum $\langle\eta \mid \zeta\rangle^{-}+\langle\xi \mid \zeta\rangle_{+}$. We shall call such Brownian algebra $\mathfrak{b}=\mathbb{C} \oplus \mathcal{G}$ the quantum (if $\|\cdot\|_{+} \neq\|\cdot\|^{-}$) Wiener $\mathrm{B}^{*}$-algebra associated with the space $\mathcal{G}$.

In the opposite case, if $\mathcal{D D}=\{\zeta \eta: \zeta, \eta \in \mathcal{D}\}$ is dense in $\mathcal{D}$, it has nondegenerated operator representation $\mathcal{C}$ on $\mathcal{D}$. Any closed involutive sub-algebra $\mathcal{E} \subseteq \mathcal{D}$ which is nondegenerated on $\mathcal{E}$ defines an Itô $\mathrm{B}^{*}$-algebra $\mathfrak{c}=\mathbb{C} \oplus \mathcal{E}$ of thermal Lévy motion. We shall call such Itô algebra the quantum (if $\mathcal{E}$ is non-commutative) Poisson $\mathrm{B}^{*}$-algebra.

In next section the general thermal algebra $\mathfrak{a}$, characterized as an Itô $\mathrm{B}^{*}$-algebra with the condition of closability of the involution $a \mapsto a^{\star}$ w.r.t. any of left or right Hilbert semi-norms on $\mathfrak{a}$, is represented as a concrete thermal $\mathrm{B}^{*}$-algebra $\mathbb{C} \oplus \mathcal{D}$.

Theorem 2. Every thermal Itô $B^{*}$-algebra is an orthogonal sum $\mathfrak{a}=\mathfrak{b}+\mathfrak{c}, \mathfrak{b} c=\{0\}$ of the Wiener $B^{*}$-algebra $\mathfrak{b}$ and the Poisson $B^{*}$-algebra $\mathfrak{c}$.

Pr o of. The orthogonal decomposition $a=\alpha \theta+b+c$ for all $a=(\alpha, \xi) \in \mathfrak{a}$, uniquely given by the decomposition $\xi=\eta+\zeta$ w.r.t. any of two scalar products in $\mathcal{D}$, where $\eta=P \xi=\xi P$ is the orthogonal projection onto $\mathcal{G} \perp \mathcal{D D}$ w.r.t. any of two Hilbert norms, and $\zeta=\xi-\eta$. 
Indeed, if $\xi \in \mathcal{D}$ is left orthogonal to $\mathcal{D D}$, then it is also right orthogonal to $\mathcal{D} \mathcal{D}$ and vice versa:

$$
\begin{aligned}
& \left\langle\eta \zeta^{\star} \mid \xi\right\rangle^{-}=\left\langle\zeta \eta^{\star} \mid \xi^{\star}\right\rangle_{+}=\left\langle\xi \mid \eta^{\sharp \star} \zeta^{b}\right\rangle_{+}=0, \quad \forall \eta \in \mathcal{D}^{-}, \zeta \in \mathcal{D}_{+}, \\
& \left\langle\eta^{\star} \zeta \mid \xi\right\rangle_{+}=\left\langle\zeta^{\star} \eta \mid \xi^{\star}\right\rangle^{-}=\left\langle\xi \mid \eta^{\sharp} \zeta^{\star \star}\right\rangle^{-}=0, \quad \forall \eta \in \mathcal{D}^{-}, \zeta \in \mathcal{D}_{+} .
\end{aligned}
$$

From these and (10) equations it follows that $\eta \xi=0=\xi \zeta$ for all $\zeta, \eta \in \mathcal{D}$ if $\xi$ is (right or left) orthogonal to $\mathcal{D D}$, and so $\|\xi\|=0$ for such $\xi$ and vice versa. Thus the orthogonal subspace is the space $\mathcal{G}=\{\xi \in \mathcal{D}:\|\xi\|=0\}$ is jointly complete w.r.t. only two norms $\|\cdot\|^{-},\|\cdot\|_{+}$and can be considered as the Hilbert subspace of pre-Hilbert space $\mathcal{D}$ with isometric involution $\xi \mapsto \xi^{\star}$ w.r.t. $\langle\eta \mid \zeta\rangle^{-}+\langle\zeta \mid \eta\rangle_{+}$. Denoting by $P$ the orthogonal projector in $\mathcal{D}$ onto $\mathcal{G} \subseteq \mathcal{D}$ we obtain $P \xi=\eta=\xi P$ and $\zeta=\xi-\eta \in \mathcal{D}$ is in the closure $\mathcal{E} \subseteq \mathcal{D}$ of $\mathcal{D} \mathcal{D}$.

4. Decomposition of Itô $\mathbf{B}^{*}$-algebras. Now we shall consider the general case. Let $\mathfrak{a}$ be an associative infinite-dimensional complex algebra with involution $b^{\star}=a \in$ $\mathfrak{a}, \forall b=a^{\star}$ which is defined by the properties

$$
\left(a^{\star} a\right)^{\star}=a^{\star} a, \quad\left(\sum \lambda_{i} b_{i}\right)^{\star}=\sum \bar{\lambda}_{i} b_{i}^{\star}, \quad \forall b_{i} \in \mathfrak{a}, \lambda_{i} \in \mathbb{C} .
$$

We shall suppose that this algebra is a normed space with respect to four semi-norms $\|\cdot\|_{\nu}^{\mu}$, indexed as

$$
\|\cdot\|_{\bullet} \equiv\|\cdot\|, \quad\|\cdot\|_{+}^{\bullet} \equiv\|\cdot\|_{+}, \quad\|\cdot\|_{\bullet}^{-} \equiv\|\cdot\|^{-}, \quad\|\cdot\|_{+}^{-}
$$

by $\mu=-, \bullet, \nu=+, \bullet$, satisfying the following conditions

$$
\begin{gathered}
\left\|b^{\star}\right\|=\|b\|, \quad\left\|b^{\star}\right\|_{+}=\|b\|^{-}, \quad\left\|b^{\star}\right\|_{+}^{-}=\|b\|_{+}^{-}, \\
\left(\|a c\|_{\nu}^{\mu} \leq\|a\|_{\bullet}^{\mu}\|c\|_{\nu}^{\bullet},\right)_{\nu=-,}^{\mu=-,} \quad \forall a, b, c \in \mathfrak{a} .
\end{gathered}
$$

Thus the semi-norms (15) separate $\mathfrak{a}$ in the sense

$$
\|a\|=\|a\|_{+}=\|a\|^{-}=\|a\|_{+}^{-}=0 \quad \Rightarrow \quad a=0,
$$

and the product $(a, c) \mapsto a c$ with involution $\star$ are uniformly continuous in the induced topology due to (17).

If $\mathfrak{a}$ is a $*$-algebra equipped with a linear positive $*$-functional $l$ such that

$$
l(a)=l(a c)=l\left(c^{\prime} a\right)=l\left(c^{\prime} a c\right)=0, \forall c, c^{\prime} \in \mathfrak{a} \quad \Rightarrow \quad a=0,
$$

and it is bounded with respect to $l$ in the sense

$$
\|a\|=\sup \left\{\left\|c^{\prime} a c\right\|_{+}^{-} /\left\|c^{\prime}\right\|^{-}\|c\|_{+}: c^{\prime}, c \in \mathfrak{a}\right\}<\infty \quad \forall a \in \mathfrak{a},
$$

where $\|a\|_{+}^{-}=|l(a)|, \quad\|a\|^{-}=\left(l\left(a a^{\star}\right)\right)^{1 / 2}, \quad\|a\|_{+}=\left(l\left(a^{\star} a\right)\right)^{1 / 2}$, then it is four-normed in the above sense. The defined by $l$ semi-norms $\|\cdot\|_{\nu}^{\mu}$ are obviously separating, satisfy the inequalities (17), and they satisfy also the $\star$-equalities of the following definition

Definition 1. An associative four-normed $\star$-algebra $\mathfrak{a}$ is called a $\mathrm{B}^{*}$-algebra if it is complete in the uniform topology, induced by the semi-norms $\left(\|a\|_{\nu}^{\mu}\right)_{\nu=+, \bullet}^{\mu=-,}$, satisfying the following equalities

$$
\left\|a^{\star} a\right\|=\left\|a^{\star}\right\|\|a\|, \quad\left\|a^{\star} a\right\|_{+}^{-}=\left\|a^{\star}\right\|^{-}\|a\|_{+} \quad \forall a \in \mathfrak{a} .
$$

The Itô $\mathrm{B}^{*}$-algebra is a $\mathrm{B}^{*}$-algebra with self-adjoint annihilator $\theta=\theta^{\star}, a \theta=0=\theta a$, 
$\forall a \in \mathfrak{a}$ called death for $\mathfrak{a}$, and the semi-norms (15) given by a linear positive $*$-functional $l\left(a^{\star}\right)=l(a)^{*}, l\left(a^{\star} a\right) \geq 0, \forall a \in \mathfrak{a}$ normalized as $l(\theta)=1$.

Obviously, any $\mathrm{C}^{*}$-algebra can be considered as a $\mathrm{B}^{*}$-algebra in the above sense with three trivial semi-norms $\|a\|_{+}^{-}=\|a\|^{-}=\|a\|_{+}=0, \forall a \in \mathfrak{a}$. Moreover, as it follows from the inequalities (17) for $c=1$, every unital $\mathrm{B}^{*}$-algebra is a $\mathrm{C}^{*}$-algebra, the three nontrivial semi-norms on which might be given by a state $l$, normalized as $l(1)=\|1\|_{+}^{-}$. However, if a $\mathrm{B}^{*}$-algebra $\mathfrak{a}$ contains only approximative identity $e_{i} \neq \rightarrow 1$, and $\left\|e_{i}\right\|_{+}^{-} \longrightarrow \infty$, it is a proper dense sub-algebra of its $\mathrm{C}^{*}$-algebraic completion w.r.t. the norm $\|\cdot\|$.

Note that $\mathrm{B}^{*}$-algebra is an old name for the $\mathrm{C}^{*}$-algebra, so the use of the obsolete term in a slightly more general sense is not contradictive. Moreover, the term Itô $\mathrm{B}^{*}$-algebra, or more appropriately, $\mathrm{B}^{*}$-Itô algebra will never make a confusion, as there is no Itô algebra which is simultaneously a $\mathrm{C}^{*}$-algebra. Indeed, suppose that an Itô $\mathrm{B}^{*}$-algebra is a $\mathrm{C}^{*}$-algebra. Then the functional $l$ is normalized to $1=l(\theta)$ on $\theta=0$, the only annihilator $\theta \in \mathfrak{a}$ in a $\mathrm{C}^{*}$-algebra, as from $\|\theta\|^{2}=\|\theta \theta\|=\|0\|=0$ it follows that $\theta=0$ in any $\mathrm{C}^{*}$-algebra. But the condition $l(0)=1$ contradicts to the linearity of $l$.

It was proved in $[3,4]$ as mentioned already in the introduction, every Itô $\mathrm{B}^{*}$-algebra $\mathfrak{a}$ is algebraically and isometrically isomorphic to a closed subalgebra of the vacuum Itô $\mathrm{B}^{*}$-algebra $\mathfrak{b}(\mathcal{H})$ of a Hilbert space $\mathcal{H}$ by $\boldsymbol{i}(a)=(\alpha, \xi, A)$, where

$$
\alpha=l(a), \quad \xi=k(a) \oplus k^{*}(a), \quad A=i(a) .
$$

It is a vacuum $\mathrm{B}^{*}$-algebra: $\mathcal{K}=\mathcal{K}_{+} \oplus \mathcal{K}^{-}$, where $\mathcal{K}_{+}=k(\mathfrak{a}), \mathcal{K}^{-}=k^{*}(\mathfrak{a})$ iff from orthogonality of $k^{*}(a)$ to all $k^{*}(c)$ with $k(c)=0$ it follows $k^{*}(a)=0$. This can be expressed as $\mathfrak{k}_{+}=\mathfrak{n}^{-}$or $\mathfrak{k}^{-}=\mathfrak{n}_{+}$in terms of the right orthogonal complement $\mathfrak{k}_{+}=\mathfrak{n}_{+}^{\perp}$ and

$$
\mathfrak{k}^{-}=\left\{a \in \mathfrak{a}:\langle a \mid b\rangle_{+}=0, \forall b \in \mathfrak{n}^{-}\right\}=\mathfrak{k}_{+}^{\star}
$$

and the ideals (9), as $\mathfrak{n}_{+}=k^{-1}(0)$ is the (left) null ideal for the map $k$ and $\mathfrak{n}^{-}=\mathfrak{n}_{+}^{\star}$. It follows from the canonical construction of $\mathcal{K}^{-}=\mathcal{K}_{+}^{*}$ as the quotient space $\mathfrak{a} / \mathfrak{n}^{-}$.

Due to the orthogonality of $\mathfrak{k}_{+}$and $\mathfrak{k}^{-}$in vacuum Itô algebras, the involution $\star$ is never defined in $\mathfrak{k}_{+}$or in $\mathfrak{k}^{-}$except on the jointly null ideal $\mathfrak{n}^{-} \cap \mathfrak{n}_{+}$. The thermal $\mathrm{B}^{*}$-algebras have the trivial ideals $\mathfrak{n}_{+}=\mathbb{C} \theta=\mathfrak{n}^{-}$, and so the involution $\star$ is defined into $\mathfrak{k}_{+}$on the whole $\mathfrak{k}_{+}=\mathfrak{a}=\mathfrak{k}^{-}$, and thus on the pre-Hilbert space $\mathcal{D}=\mathfrak{a} / \mathbb{C} \theta$ identified with $\{\xi=a-l(a) \theta: a \in \mathfrak{a}\}$, by $\xi^{\star}=a^{\star}-l\left(a^{\star}\right) \theta$. If this involution is left or right isometric (the case of tracial Itô algebras), it coincides with the left and right adjoint involutions (11). If it is not isometric, it has densely defined left and right adjoints iff it is left or right closable on $\mathcal{D}$. Thus the general thermal $\mathrm{B}^{*}$-algebra can be characterized as an Itô $\mathrm{B}^{*}$-algebra with closable involution w.r.t. the Hilbert norms on $\mathcal{D}$.

In the general case the involution $\star$ has the range in $\mathfrak{k}_{+}$only if it is restricted to the invariant domain $\mathfrak{d}=\mathfrak{k}^{-} \cap \mathfrak{k}_{+}$. Thus we can say that it is closable in $\mathfrak{k}_{+}$if $\left\|a_{n}\right\|_{+} \longrightarrow 0 \Rightarrow$ $\left\|a_{n}\right\|^{-} \longrightarrow 0$ for any fundamental w.r.t. both Hilbert semi-norms sequence $a_{n} \in \mathfrak{d}$ and so it is also closable in $\mathfrak{k}^{-}$. We shall call an Itô $\mathrm{B}^{*}$-algebra $\mathfrak{a}$ with a closable in this sense involution the $\mathrm{B}^{*}$-Ito algebra, or the general Brownian algebra if the semi-norm $\|\cdot\|=0$ is trivial on $\mathfrak{a}$, and the general Lévy algebra in the opposite case, when $\mathfrak{a} a$ is dense in $\mathfrak{a}$ w.r.t. any of the Hilbert semi-norms $\|\cdot\|^{-},\|\cdot\|_{+}$(it is always dense w.r.t. the operator semi-norm $\|\cdot\|)$. 
TheOREM 3. Let $\mathfrak{a}$ be an Itô $B^{*}$-algebra in which the restricted involution $\star: \mathfrak{d} \rightarrow \mathfrak{a}$ on the orthogonal complement $\mathfrak{d}=\left(\mathfrak{n}^{-} \cup \mathfrak{n}_{+}\right)^{\perp}$ to (9) w.r.t. both Hilbert semi-norms is left (or right) closable. Then it is an orthogonal sum $\mathfrak{b}+\mathfrak{c}, \mathfrak{b} c=0$ of a quantum Brownian $B^{*}$-algebra $\mathfrak{b}$ and a quantum Lévy $B^{*}$-algebra $\mathfrak{c}$.

Pr o of. Here we give the proof under a stronger than closability condition when the quotient algebra $\mathfrak{a} / \mathfrak{n}$ with respect to the null $\star$-ideal $\mathfrak{n}=\{a \in \mathfrak{a}:\|a\|=0\}$ has an identity, which defines the supporting ortho-projector $E=i(e)$ for operator representation $\mathcal{A}=$ $i(\mathfrak{a}) \simeq \mathfrak{a} / \mathfrak{n}$ on a Hilbert space $\mathcal{H}$. This means that there exists an element $e=e^{\star} \in \mathfrak{a}$ such that $i(a e)=i(a)=i(e a)$ for all $a \in \mathfrak{a}$,

$$
k(a e c)=i(a e) k(c)=k(a c), \quad k^{*}(a e c)=k^{*}(c) i(e a)=k^{*}(a c),
$$

and so $a e c=a c-l(a c-a e c) \theta$ for all $a, c \in \mathfrak{a}$. as it is in the canonical representation (2). We assume that $e$ is an idempotent, otherwise it should be replaced by $e^{2}$.

Under this condition, which is automatically fulfilled in the finite dimensional case, we can easily define the required orthogonal decomposition $\mathfrak{a}=\mathfrak{b}+\mathfrak{c}$ by

$$
a=b+c, \quad c=a e+e a-e a e .
$$

Here $b$ is an element of the quantum Brownian algebra $\mathfrak{b}=\{b \in \mathfrak{a}: b e=0\} \subseteq \mathfrak{n}$ which is closed in $\mathfrak{a}$ and is orthogonal to the subalgebra $\mathfrak{a} a$ as

$$
b a=b e a+l(b a-b e a) \theta=l(b a) \theta, \quad a b=a e b+l(a b-a e b) \theta=l(a b) \theta
$$

for all $b \in \mathfrak{b}$, and $b a a^{\star}=0=a^{\star} a b$ for all $a \in \mathfrak{a}$. And $c$ is an element of a quantum Lévy $\mathrm{B}^{*}$-algebra $\mathfrak{c}$, the closure of $\mathfrak{a} a$ in $\mathfrak{a}$ which coincides with all the combinations $c$ as

$$
c^{\star} c=a^{\star} a+l\left(c^{\star} c-a^{\star} a\right) \theta, \quad \forall a \in \mathfrak{a} .
$$

Thus $a=b+c, b c=0$ for all $a \in \mathfrak{a}$, where $b \in \mathfrak{b}$ is in a Brownian B*-algebra with the fundamental representation $\mathfrak{b} \subseteq \mathbb{C} \oplus \mathcal{G}_{+} \oplus \mathcal{G}^{-}$, where $\mathcal{G}_{+}=P k(\mathfrak{a}), P=I-E$, $\mathcal{G}^{-}=k^{*}(\mathfrak{a}) P$, and $c \in \mathfrak{c}$ is in a Lévy $\mathrm{B}^{*}$-algebra, having the fundamental representation $\mathfrak{c} \subseteq \mathbb{C} \oplus \mathcal{E}_{+} \oplus \mathcal{E}^{-} \oplus \mathcal{A}$ with non-degenerated operator algebra $\mathcal{A}=i(\mathfrak{a})$, left and right represented on $\mathcal{E}_{+}=E k(\mathfrak{a})$ and $\mathcal{E}^{-}=k^{*}(\mathfrak{a}) E$.

\section{References}

[1] R. L. Hudson, and K. R. Parthasarathy, Quantum Itô's Formula and Stochastic Evolution, Commun. Math. Phys. 93 (1984), 301-323.

[2] V. P. Belavkin, A new Form and ᄎ-algebraic Structure of Quantum Stochastic Integrals in Fock Space, Rendiconti del Seminario Matematico e Fisico di Milano, Vol LVIII (1988), 177-193.

[3] V. P. Belavkin, Chaotic States and Stochastic Integration in Quantum Systems, Russian Math. Surveys 47(1) (1992), 47-106.

[4] V. P. Belavkin, Representations of $\star$-semigroups Associated with Infinitely Divisible States, Quantum Probability and Related Topics, Vol VII (1992), 31-50.

[5] M. Takesaki, J. Funct. Anal. 9 (1972), 306. 